\title{
El Instituto Nacional Indigenista En EL MUNiCipio DE OXChUC, 1951-1971
}

\author{
The Mexican National Indigenous Affairs Institute in Oxchuc, 1951-1971 \\ Laurent Corbeil
}

Resumen: Este artículo analiza las relaciones entre el Instituto Nacional Indigenista (INI) y los tseltales del municipio de Oxchuc, en el contexto de un intento de integración nacional de los indígenas. El estudio de los informes indigenistas y de las comunicaciones entre ambos grupos proporciona una perspectiva más abierta y matizada sobre un proyecto que ha sido criticado. Aunque el objetivo principal de los oxchuqueros de desarrollar su economía regional no tuvo éxito, se creó una cierta integración institucional, jurídica y educacional. A pesar de los impactos negativos de tal integración, pensamos que la apertura de ambas partes permitió el establecimiento de un diálogo positivo.

Palabras clave: indigenismo, indígenas, tseltales, nacionalismo, instituciones.

Abstract: This article analyzes the relationship between the National Indigenist Institute (INI) and the Tseltals of the municipality of Oxchuc, Chiapas, in the context of the Mexican state's attempts to integrate indigenous peoples into the nation. The study of reports from the INI and of the correspondence between both groups gives a perspective more open and nuanced on a project that often has been criticized. Even though the principal objective of the Oxchuqueros of developing the regional economy was not a success, there emerged forms of institutional, juridical, and educational integration. Notwithstanding the negative impacts of such integration, this study unscores the openness of both parties that led to the establishment of a positive dialogue.

Keywords: indigenism, indigenous peoples, Tseltals, nationalism, institutions.

Laurent Corbeil, maestro por la Universidad de Montreal, candidato a doctor por la McGill University. Temas de especialización: etnohistoria, historia de México, historia colonial, historia del siglo XX. Correo electrónico: laurent.corbeil@mail.mcgill.ca.
Enviado a dictamen: 28 de marzo de 2012

Aprobación: 25 de agosto de 2012

Revisiones: 1 
A l término de la Revolución mexicana, el gobierno federal intentó establecer su legitimidad uniendo a todos los grupos sociales en el territorio para construir una identidad basada en la unidad nacional. Según los intelectuales indigenistas de la época, los indígenas representaban la parte de la población con mayor dificultad para integrarse a la nación mexicana, principalmente debido a sus escasos conocimientos de la lengua española y de la cultura social, económica y política del país (Caso, 1955: 1416). La Secretaría de Educación Pública (SEP) inició sus actividades de incorporación de los indígenas en 1921, pero los numerosos fracasos que presentaba en 1930 abrieron el camino a una serie de estudios sobre los indígenas de todo el país (Loyo, 2006: 69-94). En Chiapas, la educación socialista e indigenista de los años treinta atrajo a una pequeña porción de la población - algunas personas llegaron a ser escribanos, y así gente de poder dentro de su comunidad-, pero no logró integrar a los indígenas a la nación (Lewis, 2005: XIII-XVIII). Mientras tanto, el gobierno de Cárdenas movilizó, de 1936 a 1940, a muchos indígenas en sus tres grandes corporaciones: el Partido Nacional Revolucionario (PNR), la Confederación Nacional Campesina (CNC) y el Sindicato de Trabajadores Indígenas (STI). Estas corporaciones tenían el mandato de integrar a los indígenas en sus filas de acuerdo a sus respectivas prerrogativas, es decir, la política, la agricultura y el trabajo obrero. Esta intervención tuvo un impacto importante en algunos municipios de Los Altos de Chiapas como Chamula y Zinacantán, donde surgió una nueva élite que se apoyó en estas corporaciones para asentar su poder. Sin embargo, en otros municipios, como en el de Oxchuc, la respuesta fue más reticente (Rus, 1994: 272-280). Con el fin de superar este fracaso parcial, en la década de los años cuarenta el gobierno mexicano, siguiendo las transformaciones de la ideología indigenista, fundó en 1948 el Instituto Nacional Indigenista (INI). Fundado en 1950 en San Cristóbal de Las Casas, el Centro de Coordinación
Indigenista Tzeltal-Tzotzil (CCITT, también llamado en adelante "el Centro") representa el mayor intento de escolarización de la población indígena en la región (Rockwell, 2006: 63). Sus actividades comenzaron en 1951 y fueron particularmente importantes en una docena de municipios de Los Altos hasta 1971, cuando la Dirección General de Educación Extraescolar en el Medio Indígena reemplazó al INI en el ámbito de la educación (Pineda, 2004: 287).

Por lo general, los historiadores han estudiado el impacto del indigenismo en el conjunto de Los Altos y no en comunidades específicas, demostrando que la integración de los indígenas ha sido, a fin de cuentas, negativa. Según Henri Favre (1971: 327-330), el proyecto del INI no funcionó ya que ambas culturas, indígena y ladina, eran incompatibles $y$, por lo tanto, no se podía llevar a cabo ninguna transformación cultural ni estructural. Como el INI había luchado sólo en contra de los problemas internos de las comunidades, no logró cambiar las estructuras externas ni liberar a ese pueblo oprimido. Nancy Modiano (1974: 179-192 y 252-253) y Ulrich Köhler (1975: 329-330) testifican, por su parte, un éxito parcial del programa debido principalmente a factores externos a los indígenas. Para Modiano, los únicos que pudieron imaginar el país de manera más completa fueron los que continuaron sus estudios fuera de las comunidades en las escuelas más avanzadas. Para Köhler, la aculturación fue el resultado de factores independientes tales como la distancia entre San Cristóbal y los municipios, y la presencia de misioneros evangélicos que habrían preparado el terreno para la llegada del INI. Así, para estos autores, los indígenas no hicieron más que recibir las influencias de la población ladina. En un análisis comparativo de cuatro monografías históricas sobre las reformas culturales posrevolucionarias en Chiapas, Robert Carmack (1989: 401-425) sostuvo que los indios son percibidos por los historiadores como seres pasivos que han sufrido transformaciones sin reaccionar. ${ }^{1}$ Más recientemente, Elsie Rockwell (2006: 67-68) argumentó 
que se debe considerar la participación indígena en el establecimiento y funcionamiento de un programa educativo, puesto que varios factores internos - como las relaciones internas de poder, las condiciones de vida y las definiciones de la identidad personal, comunitaria, cívica, étnica y nacional - también han sido responsables de varios cambios en el modo de vida.

Creemos que esta última interpretación se debe considerar seriamente en el estudio de la intervención del INI. De hecho, a su llegada a Los Altos de Chiapas, el Instituto se percató de que el sistema de educación que le había precedido tenía varios defectos puesto que no fomentaba la participación local. Para remediar esta situación, el INI solicitó el apoyo de algunos padres de familia y de las comisiones de educación locales, tanto para el desarrollo del programa de educación, como para la resolución de los problemas escolares (Köhler, 1975: 189-193; Modiano, 1974: 182). Además, de acuerdo con Edmundo Henríquez Arellano (2000: 29-60) y Luz Olivia Pineda (1993: 82-90), pareciera que los esfuerzos del INI contribuyeron a que algunos promotores culturales se convirtieran en los representantes políticos de sus municipios. Pineda aún habla de la creación, entre 1940 y 1970, de una nueva "burguesía indígena". En consecuencia, el estudio de las dinámicas internas en las comunidades indígenas se presenta como un aspecto esencial para comprender las actividades del INI en Los Altos de Chiapas.

Con el objetivo de entender mejor estas dinámicas, estudiaremos el programa del INI en un solo municipio. Situado a unos 30 kilómetros al noreste de San Cristóbal y con 5412 habitantes, casi exclusivamente tseltales en 1950 (Romano, 2002: 278-279; Séptimo censo de población, 1950), Oxchuc ha sido el municipio que mostró mayor entusiasmo por el programa. De hecho, desde 1951 hasta 1968 los oxchuqueros representaron al menos el 25\% de todos los promotores en la región ${ }^{2}$ y el número de escuelas pasó de ocho en 1952 a 21 en $1963 .{ }^{3}$ El historiador Ulrich Köhler (1975: 210) señaló también que los jóvenes estuvieron especialmente motivados para trabajar con el INI ya que querían transformar su sociedad, adaptarla al nuevo marco mexicano y abrirla a otras posibilidades que no estuvieran relacionadas exclusivamente con la agricultura de subsistencia. Tal nivel de participación podría tener su origen en la situación de pobreza que prevaleció en el municipio antes de la llegada del INI. Según el censo de 1950, la mayoría de la población vivía en casas de madera (90.5\%) y caminaba descalza (93.4\%). Sólo un 15\% sabía leer y escribir. La ausencia de comunidades concentradas hacía difícil el acceso a recursos esenciales como el agua, la asistencia médica y el comercio. Además, Oxchuc tenía la mayor densidad poblacional en la región, presentaba una distribución de la tierra muy desigual y estaba dividido, desde la década de 1940, en dos ámbitos religiosos: el evangélico en el norte y el católico en el sur. ${ }^{4}$ Esta situación causó graves conflictos de tierras, muchas tensiones políticas y problemas religiosos frecuentes.

En este artículo sostenemos que la situación socioeconómica de los oxchuqueros permitió un éxito parcial -insistimos en la palabra parcial- del INI. En primer lugar mostramos que, mientras que para el Instituto el principal objetivo fue la integración, sobre todo cultural, para los indígenas la prioridad fue mejorar su situación social y desarrollar la economía regional. En seguida subrayamos que esta discrepancia de prioridades no impidió por completo la integración nacional. De hecho, se construyó un acuerdo entre los dos grupos para que se creara una integración institucional, jurídica y educacional. Ahora bien, ningún grupo humano es homogéneo. Sin embargo, creemos que, si buscamos una aportación positiva al programa, la difusión de las normas y de las instituciones nacionales, así como su utilización por unos oxchuqueros, fue la mayor contribución del INI al municipio indígena.

Como este estudio de caso es un estudio histórico, trabajamos sobre todo con fuentes escritas. Las interacciones entre los indígenas y el CCITT se dieron sobre todo a través de las autoridades municipales, los comités de educación y los promotores culturales por 
un lado y, por el otro, a través de los inspectores. Así, la mayor parte de las fuentes históricas que encontramos son informes redactados por estos últimos como agentes del INI. De vez en cuando, los promotores o las autoridades municipales se comunicaban por escrito con el director de educación del Centro, lo que resulta ser una fuente muy interesante, aunque no tan frecuente. No obstante, podemos inferir los deseos y los comportamientos de la población de Oxchuc comparando estos dos tipos de fuentes. ${ }^{5}$

\section{Percepciones de la educación indigenista en Oxchuc}

Uno de los objetivos principales del indigenismo fue encaminar a los indígenas a redefinir por ellos mismos su identidad con el fin de encarnar al mexicano mestizo, representante por excelencia tanto del patrimonio americano como de la modernidad occidental. Debido a sus recursos financieros limitados (Köhler, 1975: 168; Favre, 1996: 102-104), el Instituto tuvo que apoyar su programa en varios actores sociales indígenas como los promotores culturales - que tal vez representaron la mayor aportación del INI en comparación con la educación federal y estatal- ${ }^{6}$ los miembros de los comités de educación, los padres, los alumnos y los ciudadanos locales. Así, los indígenas no sólo fueron el objetivo de los indigenistas, sino también la herramienta principal de su proyecto.

Esta integración nacional se intentó mediante varios elementos de la vida cotidiana. El mejoramiento de la higiene y la salud estaba en parte destinado a transformar la mala imagen del "indio", cuyo calificativo refería muchas veces a la imagen de una persona sucia, llena de enfermedades y de parásitos, de piel oscura cubierta de tierra, entre otros estereotipos. De hecho, el INI intentó mejorar elementos relacionados con la apariencia tales como el pelo bien cortado y peinado, la limpieza de las viviendas y la lucha contra la promiscuidad, considerados todos elementos esenciales para mejorar la calidad de vida, al mismo tiempo que definían la pertenencia a los niveles más altos de la jerarquía socio-étnica. Los promotores también debían asegurarse de que las infraestructuras comunitarias reflejaran la modernidad de la nación; incluso, tenían que cuidar que las escuelas fueran construidas de piedra, con ventanas y puertas suministradas por el Centro, a fin de establecer el ejemplo a seguir para la construcción de cualquier otro edificio en el paraje. Además, la escuela formaba, junto con el monumento a la bandera mexicana, el centro de la comunidad modernizada en donde el promotor y sus alumnos tenían que cantar el himno nacional cada mañana. A su vez, la intención de cambiar los modos de producción agrícola reflejaba el deseo de establecer, en esta parte remota del país, un modo de producción capitalista. En conformidad con las normas de desarrollo económico nacional, los promotores enseñaban técnicas modernas que supuestamente habrían permitido la producción de excedentes agrícolas, así como el establecimiento de mercados locales relacionados con los principales centros comerciales. Asimismo, las capacitaciones que abarcaban a todos los promotores de la región y las visitas a otros municipios tenían como propósito el intercambio de ideas sobre el desarrollo y la homogeneización de los grupos presentes. Por último, mediante la enseñanza escolar los promotores debían favorecer un mejor conocimiento de los derechos y deberes de los ciudadanos mexicanos, así como la difusión de la lengua nacional con el fin de que se convirtiera en la más importante. En su conjunto, el trabajo de los promotores estaba destinado a aumentar en la conciencia de los oxchuqueros la idea de que pertenecían a la moderna nación mexicana.

Con el fin de que los promotores fueran capaces de cumplir el papel que les había asignado el INI, era necesaria la participación no sólo de los alumnos, sino también de todos los miembros de la comunidad. Si el promotor tenía el encargo de enseñar y de mostrar la dirección correcta, la responsabilidad de poner 
en práctica los conocimientos recaía siempre en la comunidad entera. Por ejemplo, una vez dadas las recomendaciones iníciales por el promotor sobre la higiene, los padres debían ser los que vigilaran y cuidaran la salud de sus hijos. Asimismo, los padres se vieron fuertemente incitados a frecuentar las clases impartidas por los promotores en la noche, a participar en las reuniones escolares y a presentarse frente a los inspectores del INI cuando pasaban por el paraje. Todo ello alentó la responsabilidad de los padres en cuanto a la educación y alfabetización de sus hijos.

Sin embargo, lo que atrajo a los oxchuqueros fueron sobre todo las promesas de mejoramiento económico. Según los informes mensuales de los inspectores del INI, la participación indígena en este municipio se concentró en la educación, en la construcción de infraestructuras, en el aprendizaje de mejores técnicas para la ganadería, la agricultura y la artesanía, y en el desarrollo de nuevas oportunidades de empleo. Así, los indígenas fueron los que construyeron las escuelas, las casas de los promotores y los campos de deportes, entre otras instalaciones. Si bien el INI exigía, antes de enviar un promotor, que un paraje presentara alrededor de 50 alumnos, que demostrara la existencia de recursos materiales y obreros suficientes, y que hubiera una gran motivación por parte de los padres, en realidad nunca pudo cumplir con todas las demandas de los indígenas en este municipio. En sólo cuatro meses de operación, en diciembre de 1951, el Centro rechazó tres demandas de escuelas sobre ocho por falta de recursos. ${ }^{7}$ En julio de 1952, el inspector Reynaldo Salvatierra escribió en su informe:

Pedro López [presidente del comité de educación del paraje de Bumiljà] y otros más hablaron repitiéndonos lo que en Oxchuc, que estaban deseosos de aprender a leer y suplicaban se les diera un maestro. Se formaron los niños [...] 32 hombres y tres mujeres; uno de los indígenas habló indicando que faltaba más de la mitad, que por todos suman 72 entre niños y niñas.
[...] Continuando nuestro camino, en el trayecto nos encontramos a varios indígenas que llevaban a sus hijos al lugar de la reunión en Bumiljá, se leía en ellos la aflicción de no haber podido llegar a tiempo porque deducían que nosotros éramos los de la visita a su paraje (AHCCITT: educación, informes, 1952, $1,0004)$.

Tales ejemplos se repitieron muchas veces en los informes de los inspectores indigenistas y en la correspondencia de las autoridades indígenas locales, por lo cual aparece de una manera muy evidente el deseo de cambio. ${ }^{8}$

El origen de tal motivación para el aprendizaje fue múltiple. Algunos deseaban mejorar su estatus en las relaciones comerciales regionales y alcanzar un nivel similar al de los ladinos, por lo que una mejor educación les parecía una buena medida. Saber leer, escribir y contar los volvía significativamente menos propensos a ser engañados y les proporcionaba un mayor poder de negociación. ${ }^{9}$ Otros tuvieron la oportunidad de seguir estudiando fuera del municipio - en San Cristóbal o en Tuxtla Gutiérrez, por ejemplo- para especializarse y practicar una profesión con mejor remuneración y prestigio, como abogados, médicos o políticos. ${ }^{10}$ Una gran proporción de la población también se interesó en las nuevas técnicas agrícolas, y algunos, como Manuel Gómez López, tomaron cursos avanzados en este campo para encontrar empleo como ingenieros agrónomos de gobierno y difundir sus conocimientos en su municipio." A veces el entusiasmo de los oxchuqueros iba aún más allá de las capacidades del Centro. Por ejemplo, la escuela abierta, evento puntual en el que los promotores impartían una breve clase sobre algún tema que conocían bien, tuvo que cesar sus operaciones por falta de dinero a pesar de su éxito rotundo. ${ }^{12}$

Tanto el proyecto de desarrollo como la motivación de los indígenas presagiaban un gran éxito del programa en el municipio. Muchos veían la educación como una oportunidad para abrirse a una mayor variedad 
de trabajos más rentables. Sin embargo, aunque muchos aprendieron nuevos oficios y técnicas de trabajo, así como nuevos métodos de negociación y de comercialización, este aprendizaje no produjo resultados convincentes. El panorama del empleo en Oxchuc cambió muy poco entre 1950 y 1970. Aunque los habitantes de Oxchuc llegaron a tener buenas nociones de escritura, matemáticas y español, y aunque muchos solicitaban el empleo de promotor, el Centro sólo podía proporcionar un número limitado de puestos. En consecuencia, muchos varones se encontraron relativamente bien educados, pero sin la posibilidad de usar sus conocimientos a cambio de un sueldo al interior del municipio. Tenían o que seguir practicando la agricultura de subsistencia, o bien salir de su comunidad. Por lo tanto, el porcentaje de habitantes de Oxchuc con puestos de trabajo en dominios distintos a la agricultura sólo aumentó de uno a 1.5\% de 1950 a 1970 (ver tabla 1).

Así, a pesar de una destacada participación, la mayoría de ellos no mejoró sus condiciones económicas durante esos años.

Podría explicarse este resultado por la gran diferencia en las interpretaciones que ambos grupos tenían de los objetivos del programa indigenista. Por supuesto, los dos grupos sabían que los objetivos eran múltiples. Sin embargo, parecería que el INI se enfocó más en su papel de agente de aculturación, mientras que los indígenas buscaban principalmente mejorar su calidad de vida a través de la diversificación de su economía y de una mejor educación. Los dos grupos trabajaron siempre juntos, aunque en el ámbito de la economía su colaboración parece haber sido un fracaso.

\section{La integración institucional}

Los habitantes de Oxchucy los indigenistas encontraron mayores afinidades en el ámbito de las instituciones nacionales, pues parece que los mayores beneficios obtenidos por los indígenas se lograron haciendo uso de ellas. Ya sea a través de la laicidad del sistema de educación, del establecimiento de los niveles nacional y local de justicia, o de la organización política del municipio, los protagonistas de ambos grupos pudieron sacar provecho de las instituciones. La mayoría de las veces este acuerdo tácito se construyó a través de la resolución de conflictos internos o regionales.

Desde su llegada a Oxchuc, el Centro participó involuntariamente en las luchas religiosas, ya que tres de sus principales escuelas - las de Cholol, Chaonil y Mesbiljá- se encontraban precisamente entre el norte evangelista y el sur católico del municipio. ${ }^{13} \mathrm{El}$ CCITT no tenía intenciones de resolver esos conflictos religiosos ni de imponerse como árbitro. Más bien optó por la postura laica del Estado nacional en las escuelas públicas para impedir cualquier predicación religiosa, evitando la presencia de objetos religiosos o la discriminación basada en la religión. Para lograr esto, el CCITT tuvo que involucrarse en la elección de ciertas autoridades educativas locales a fin de garantizar que las diferentes religiones fueran representadas en los comités locales de educación. Esto permitió a la gente seguir practicando cualquier religión, pero no llevarla al medio educacional. También, el CCITT capacitó a sus promotores poniendo énfasis en que la predicación estaba prohibida en las escuelas y que ellos debían fungir como agentes de educación para todos los indígenas, y no sólo para aquellos que practicaran una religión en particular. ${ }^{14}$

La mayoría de las disputas religiosas tuvieron lugar en el centro de la ciudad, en donde los misioneros evangélicos intentaron imponerse. El paraje de Cholol, donde había influencia de Mariana Slocum, agente del Instituto Lingüístico de Verano, una organización conocida por sus actividades misioneras evangélicas, fue el escenario de muchos conflictos religiosos. El inspector se había asegurado de que el comité de educación estuviera compuesto por tres evangélicos y tres católicos para que no se tomaran decisiones basadas en la fe ni hubiera algún tipo de discriminación religiosa, 
ya fuera frente a los niños o frente a las oportunidades de desarrollo. Sin embargo, el promotor Daniel Gómez Rodríguez consiguió que en marzo de 1952 su escuela fuera exclusivamente evangélica, bloqueando el acceso a todos los niños católicos. El inspector pudo convencer al promotor de que cumpliera con la laicidad del sistema educativo mexicano, pero en 1956 predicadores evangélicos interrumpieron las actividades de la escuela con el propósito de reafirmar su poder sobre los demás habitantes del paraje. Se enfrentaron entonces no sólo al INI, sino también al nuevo promotor Feliciano López Gómez ${ }^{15}$ yal presidentemunicipal, quienes restablecieron el orden. El carácter laico de la institución mexicana ya se había convertido en norma en Oxchuc y las autoridades lo aplicaban de manera uniforme. ${ }^{16}$ En otras partes las disputas religiosas fueron menos recurrentes, lo que permitió al Centro resolver con mayor facilidad los conflictos. Si la predicación religiosa podía ser una herramienta de lucha poderosa para aquellos que querían elevar su condición social, su potestad se desmoronó frente a los poderes que el sistema de educación y el Estado nacional conferían a los ciudadanos. De hecho, el último conflicto religioso que encontramos en los archivos del CCITT tuvo lugar en 1959. ${ }^{17}$

Por lo general, el Centro prefería no intervenir directamente en las disputas internas del municipio, a menudo relacionadas con las condiciones de vida difíciles y el contexto político tenso porque creía que el orden social debía ser establecido por los propios indígenas. Sobre todo buscaba promover, mediante la educación, las normas legales de México. Así, cuando un inspector encontraba un problema entre individuos, la mayoría de las veces lo refería a las autoridades municipales haciendo algunas recomendaciones, pero procurando no juzgar el caso de antemano. De esta manera, cuando era posible, el INI sólo intervenía como intermediario y dejaba a las personas resolver sus propios problemas. Sin embargo, el Instituto fue capaz de afirmar sutilmente la competencia superior de la justicia y ley nacionales. De hecho, las autoridades de Oxchuc, a diferencia de las de otros municipios, utilizaron al INI para legitimar y establecer su poder, de tal manera que incorporaron las normas del sistema político-legal mexicano.

Este repartimiento de las competencias jurisdiccionales entre los órganos estatales y municipales se manifestó poco después de la llegada del INI. En abril de 1954, el inspector informó al presidente municipal de un problema jurídico relativo a la venta de tierras. La familia López Kaná había comprado tierras de Remigio Néjera, quien no había cumplido con las normas de ventas al no proporcionar ninguna documentación que acreditara la transacción. En este caso, el inspector sostuvo que trabajó con el presidente municipal para resolver el problema, dejándolo tomar las medidas necesarias aunque asegurándose de que se respetara el proceso legal. ${ }^{18}$

En las comunidades donde intervino el INI, la figura de autoridad local, aún superior al comité de educación, parece haber sido la del promotor, cuyo cargo cumplía con la labor de vigilar la estabilidad de su comunidad. Podían surgir algunos conflictos cuando, por ejemplo, unos indígenas con más tierras que otros aceptaban dar una parte de su propiedad para la construcción de infraestructuras relacionadas con el programa del INI. Algunos vecinos, ambiciosos de posesiones, quisieron apropiarse más de lo debido. Tal fue el caso en Chaonil en abril de 1952, cuando Jacinto Luna concedió una buena parte de sus tierras a la construcción de una escuela del INI. Sin embargo, los vecinos del paraje decidieron tomar la superficie necesaria no sólo para la escuela, sino también para el desarrollo de todas las infraestructuras indigenistas. Cuando Jacinto Luna presentó la queja ante el inspector, éste decidió encargarle al promotor la resolución de la crisis, sugiriendo que pidiera la contribución en tierras de los demás vecinos. ${ }^{19}$ El objetivo del inspector fue resolver el problema desde el interior de la comunidad al otorgar el poder al promotor para que actuara siguiendo las normas y las leyes nacionales. 
En Oxchuc, la articulación de una política municipal enmarcada por el gobierno federal fue posible gracias al INI y a su programa de educación. De hecho, a diferencia de otros municipios de Los Altos, los protagonistas de los conflictos internos en este municipio utilizaron los diversos elementos del INI, como los puestos de promotores, de miembros de comités de educación y de especialistas, para mejorar su estatus social. Por ejemplo, algunos promotores lograron convertirse a lo largo de los años en presidentes municipales, como fue el caso de Juan Gómez Nich, que obtuvo el puesto de promotor de Tzunum en 1951. Para entonces ya había sido elegido presidente municipal un año antes, pero había construido su reputación apoyándose en su educación - aunque fuera mínima- y en su participación en el comité de educación de Yochib durante los años 1930 y 1940 (Gómez Nich, 1976: 143-146). Otros siguieron sus pasos, como Alonso Morales Sánchez en 1960, Manuel Morales Díaz en 1964, y Marcelo Sántiz López en 1968 (Morales Sánchez, 1976: 136; Morales Díaz, 1976: 115; Sántiz, 1976: 170; ver también Pineda, 1993).

De manera general, estos "promotores-presidentes" colaboraron positivamente con el INI y el programa de educación porque ello les permitió sacar provecho de esta doble experiencia. Sin embargo, el Instituto siempre tuvo que vigilar las actitudes y, de vez en cuando, tomar medidas para moderar sus excesos. Por ejemplo, en octubre de 1958 el inspector no quiso involucrarse directamente en dos conflictos internos de Oxchuc y, por lo tanto, pidió a las autoridades municipales su intervención. En el primer caso, las autoridades municipales encarcelaron por 72 horas a cuatro hombres del paraje de Pachtontikjá supuestamente por poner impedimentos para la construcción de la escuela local. No obstante, se pidió también al comité de educación que pagara a los encarcelados las horas de trabajo perdidas durante su estancia en la cárcel. Al respecto, el inspector comentó que "es una forma rara de impartir justicia [...]”, pero decidió dejar el asunto en manos de los padres de familia, que al final expulsaron a los cuatros indeseables. En el segundo caso, ante una queja de los padres de Tuxaquiljá en contra de su promotor, las autoridades municipales resolvieron el asunto de conformidad con ambas partes, pero impusieron una multa de 600 pesos al promotor para, según el inspector, atemorizarlo. Por mucho que el inspector quiso solucionar la disputa de manera interna, no pudo pasar por alto la injusticia en contra del promotor, por lo que solicitó al Centro que realizara una investigación sobre el caso. ${ }^{20}$

Lo sobresaliente en ambos casos fue que se trató de conflictos relacionados con las competencias y los poderes de cada nivel de autoridad, es decir, del Estado representado por el INI, de las autoridades municipales y de las autoridades educativas locales. Parecería que las autoridades municipales se encontraban protegidas por indigenistas pues, como hemos dicho, muchos de ellos tenían estrechos lazos con el INI. Sin embargo, para alcanzar sus objetivos el INI tuvo que vigilar también su imagen en las localidades a fin de no perder la confianza de los participantes. Según los pocos casos de conflictos que encontramos entre el INI y las autoridades, parecería que el INI logró imponer el punto de vista del gobierno y la jerarquía de autoridad deseada. Por ejemplo, durante el mandato como presidente municipal de Alonso Morales Sánchez, de 1960 a 1963, encontramos un sólo caso de abuso de poder, el cual fue considerado tan grave por el Centro que éste pidió al director general de asuntos indígenas de Chiapas que vigilara la situación. Como esto sucedió en 1960, al principio del mandato de Morales Sánchez, suponemos que no se debió a una voluntad de desafiar al INI, sino más bien a un error de inexperiencia ante el sistema político y judicial. ${ }^{21}$

Si bien los habitantes de Oxchuc lucharon a veces contra ellos como sucedió en otros municipios (Rus, 1994; Pitarch, 2004; Pineda, 1993), finalmente no rechazaron al INI, sino que lo utilizaron como instrumento social, pensando en que éste les daba el derecho. Sin embargo, por su parte, el INI veía los poderes municipales y locales con la visión del Estado 
nacional, es decir, como subordinados a los que había que imponer ciertos límites. Por lo tanto, tuvo siempre que encontrar el equilibrio entre la iniciativa de dejar a los oxchuqueros actuar por su propia voluntad, y su deber de agente de gobierno y difusor de las instituciones políticas, legislativas y judiciales. Tal equilibrio no siempre se alcanzó, y la llegada de los promotores-principales cambió la estructura de poder local y a veces incluso dio lugar a conflictos internos al municipio (Pineda, 1993). Por ejemplo, la familia del último promotor-presidente bajo el programa del INI, Marcelo Sántiz López, conserva una posición bastante poderosa en el municipio hasta hoy en día, algo que no logró sin conflictos. Sin embargo, lo positivo de la modificación de la estructura de poder fue que quebró el aislamiento que caracterizó a Oxchuc antes de 1950.

\section{El INI y la lucha indígena en contra de la opresión ladina}

Hasta ahora hemos expuesto que la apertura de los habitantes de Oxchuc al trabajo del INI y su adopción de las estructuras de poder originaron su deseo de mejoramiento económico y social. Pensamos también que uno de sus objetivos de participar tan activamente en el programa de educación indigenista fue el de transformar las relaciones de tipo colonial entre los indígenas, los mestizos y los ladinos. Dentro del contexto de las luchas étnicas y sociales que presentaba la oposición histórica entre los señores ladinos y los peones indígenas, oposición que sigue existiendo en esta "sociedad de casta" (Favre, 1971: 99-101 y 267; Womack, 1999: 4-6; Viqueira, 2002: 276-281), el INI aportó a los indígenas un apoyo muy valioso. La educación impartida por el Centro les permitió cultivar la tierra de manera más eficaz, formar comunidades donde la ayuda mutua y la defensa de los intereses comunes se daban de manera más fácil y construir infraestructuras que favorecieron el desarrollo social y económico, pero, sobre todo, aprender a leer y a escribir, herramientas esenciales para la defensa legal de sus intereses.

El paraje de Naokil representa un buen ejemplo del tipo de apoyo que prestó el INI para los indígenas de la región. Se trata de una comunidad situada en el municipio de Huixtán conformada por indígenas que vivían y trabajaban en las tierras de un finquero que les pagaba poco, o muchas veces nada, y que ejercía con frecuencia la violencia en su contra. Por ejemplo, en agosto de 1953 los propietarios ladinos de unas tierras vecinas destruyeron, en colaboración con las autoridades de Huixtán, las casas de cinco indígenas con el pretexto de que habían matado a una de sus vacas. Según el inspector Reynaldo Salvatierra, se trató de un caso de explotación pura, donde los ladinos quisieron mostrar su posición de poder y de fuerza frente a estos indígenas sin recursos. Apoyados por los consejos de los indigenistas, tuvieron que luchar legalmente para deshacerse del dominio de esos hombres y comprar unas tierras en el municipio de Oxchuc. En mayo de 1957, el asunto parecía arreglado; no obstante, surgió nuevamente meses después, cuando un mestizo de Oxchuc aconsejó al indígena que prestó sus tierras a la gente de Naokil que había que expulsarlos, pues, como recibían educación por parte del INI, podían robarle todas sus tierras. Tuvieron entonces que reubicarse de nuevo y fundaron el paraje de La Independencia, todavía en Oxchuc. ${ }^{22}$ Los eventos que experimentó la gente de Naokil demuestran que los indígenas podían encontrar en el INI un fuerte apoyo para liberarse de la opresión socio-étnica dado que el Instituto estorbaba a la gente no indígena en el uso y abuso de su poder.

Además de la explotación, la mayoría de los problemas sociales de la población de Oxchuc venía de los conflictos sobre las tierras agrícolas, que se tornaban violentos de vez en cuando. En el verano de 1955, por ejemplo, las tensiones entre los lugares vecinos del paraje indígena de Kistoljá y la finca El Retiro alcanzaron tales niveles que los indígenas amenazaron con hacer uso de la violencia en contra de los propietarios de la 
finca y de las autoridades municipales. En ese momento intervinieron la policía y el INI, la primera para evitar que hubiera peleas y, el segundo, para convencer a la gente de Kistoljá de que existían otros recursos legales para resolver sus quejas. De esta manera, los de Kistoljá decidieron presentar una querella en México en la cual dos promotores fungieron como intermediarios. A pesar de que el proceso legal fue largo, puesto que hasta mayo de 1956 aún no se había juzgado el caso, los consejos del Instituto calmaron las tensiones y terminaron con el riesgo del brote de violencia. Además, utilizando las instituciones judiciales mexicanas, los oxchuqueros demostraron su voluntad por adoptar y apoyar sus luchas en las normas legales del gobierno federal. ${ }^{23}$

El INI proporcionó a los habitantes de Oxchuc una capacitación que les permitió resolver sus conflictos socio-étnicos de manera legal, salir de la explotación y de la violencia en las que se encontraban, y entender mejor su papel en la nación mexicana. Muchos señalaron la importancia de tener un buen nivel de educación para seguir legalmente con sus luchas; incluso, la mayoría de los promotores enseñaron a los niños y a sus padres los derechos y obligaciones de los ciudadanos mexicanos, así como la Constitución de la nación mexicana y algunas leyes. ${ }^{24}$ Además, muchos parecieron haber adoptado nuevos comportamientos orientados hacia el exterior del municipio, entre los que destacaron el de viajar para seguir estudiando, empezar diligencias legales y desarrollar nuevos métodos comerciales. Como Oxchuc no había participado en las tentativas de integración nacional de los años treinta del siglo pasado, ni tampoco en las corporaciones nacionales como el PNR, el CNC y el STI, y como las instituciones nacionales le eran más accesibles a través del INI, logró integrarse a ellas con mayor eficacia que otros municipios de Los Altos. Pareciera que el enfoque educativo del INI y su flexibilidad en la aplicación de su programa permitieron una participación más integral de la población indígena y crearon en ellos una mayor confianza frente a la nación y sus instituciones.

\section{Integración al sistema de educación nacional}

Paralelamente a lo arriba expuesto, se llevó a cabo la integración de los indígenas al programa de educación nacional. Tal como se mencionó al principio, la mayoría despreciaba a los maestros de la SEP porque representaban la explotación, ya que algunos de ellos llegaron a explotar a sus alumnos haciéndoles trabajar. Sin embargo, ante la gran motivación de los oxchuqueros por el programa y ante el éxito de varios alumnos, el INI no tuvo otra opción que mejorar su programa. Por ejemplo, ya en octubre de 1952, los alumnos de Mesbiljá pedían libros más avanzados en lengua castellana y el Centro tuvo que proporcionarlos..$^{25}$ En 1956, el Centro creó una escuela de transición en la cual los estudiantes avanzados podían alcanzar un nivel de educación superior, en el contexto todavía equivalente a la escuela primaria. También, para que se pudiera utilizar dicha educación el INI quería que sus estudiantes fueran reconocidos por el sistema nacional de la SEP. ${ }^{26}$ Por último, los primeros promotores del Centro tenían un nivel de educación bastante bajo que difícilmente superaba la primaria, e incluso raras veces el tercer año. Así, muy pronto los alumnos alcanzaron los mismos niveles que sus maestros y no podían superarlos. Por tal motivo, a partir de 1960 el Centro envió a muchos de sus promotores a una escuela de Oaxaca con el fin de que llegaran a los niveles de bachillerato y profesional. ${ }^{27}$

Estas transformaciones superficiales en el programa de educación provenían de otros cambios mucho más sustanciales. Dado que el proyecto se trataba más de un ensayo con un presupuesto muy bajo que de un verdadero plan de desarrollo, el CCITT se encontró rápidamente sin recursos para satisfacer las necesidades de los indígenas. Para resolver el problema, el Centro recurrió primero al gobierno del estado de Chiapas. Así, en septiembre de 1955 el director Fidencio Montes Sánchez afirmó que existía un acuerdo entre los dos sistemas de educación para usar las mismas técnicas de 
enseñanza en todas las municipalidades indígenas del estado. Incluso, expresó la posibilidad de que el estado empleara y pagara a los promotores del Centro si éste no lo podía hacer de manera aceptable. ${ }^{28}$ En 1958 se elaboró un plan de coordinación de los sistemas federal, estatal e indigenista. ${ }^{29} \mathrm{El}$ primer paso de esta coordinación de esfuerzos educativos consistió en que la SEP aceptara que la enseñanza de primer año se proporcionara únicamente en la lengua indígena y que, por lo tanto, fuera impartida por profesores indígenas, tal como lo hacía el INI. En 1959 se dieron los primeros traslados de personal, cuando los promotores Agapito Núñez Tom y Marcelo Sántiz López se volvieron profesores rurales de clase A por la SEP. ${ }^{30}$ De esta forma, siguieron trabajando en las mismas localidades y llevando a cabo las mismas tareas que hacían para el INI, pero ya entonces les pagaba la SEP. Conforme se estableció la coordinación entre las dos instituciones y el número de alumnos aumentó, las tareas de los promotores del INI y de los profesores de la SEP se fueron distinguiendo y precisando cada vez más. En 1969 los promotores del INI sólo tenían que enseñar a los alumnos más jóvenes y monolingües, mientras que los profesores de la SEP, anteriormente promotores del INI, se encargaban de los niveles superiores de nivel primaria. ${ }^{31}$

En 1970, el CCITT todavía controlaba 133 escuelas en toda la región de Los Altos. Sin embargo, la gran mayoría de sus empleados eran maestros de la SEP, mientras que sólo siete promotores trabajaban directamente para el INI. Por el contrario, en la misma zona la SEP pasó de siete escuelas en 1965, a 115 en $1970 .{ }^{32}$ Las capacitaciones de los profesores habían cambiado bastante, pues la SEP sólo recibía a los mejores promotores del INI que tenían, al finalizar los años sesenta, la educación preparatoria terminada y, de vez en cuando, una formación de nivel profesional. Dado que estos niveles de educación los obtenían fuera de Chiapas, los profesores regresaban a sus comunidades con nuevos valores aprendidos en la esfera nacional para enseñar así a sus alumnos. De este modo, además de encontrar una solución viable y eficaz a la falta de recursos del INI, el Estado nacional favoreció la integración gradual de la educación regional al programa de educación federal. Este traslado de la educación, de lo muy regional a lo nacional, cumplió con el objetivo principal del INI, es decir, el de integrar a toda esa región indígena a la nación mexicana.

\section{Conclusión}

En el contexto político del México posrevolucionario, el Instituto Nacional Indigenista tuvo el objetivo de integrar a los indígenas a la nación mexicana llevando a cabo modificaciones culturales, económicas y políticas. Para los indigenistas, la adopción de la cultura mexicana por parte de los indígenas podía transformarlos en mexicanos. Aunque tuviera objetivos laudables para mejorar las condiciones de vida de los indígenas, el Instituto cumplió con sus deberes de manera parcial e imperfecta, en parte porque muchos municipios no quisieron participar en el programa. En Oxchuc, sin embargo, el INI tuvo un mayor éxito, primero porque supo adaptarse al contexto local y, en segundo lugar, gracias a la participación positiva de la mayoría de los oxchuqueros. Entre los deseos nacionalistas del Instituto y la búsqueda de mejores condiciones de vida por parte de los indígenas, ambos grupos protagonistas lograron llegar a acuerdos sobre la base de las normas y de las instituciones nacionales. Se puede decir con seguridad que el igualitarismo económico y jurídico entre estos indígenas y los mexicanos no se había alcanzando en 1971, por lo que se pueden presentar muchas quejas hacia el INI. También se puede añadir que la integración institucional, y sobre todo educativa, fue sin duda fuente de poder para unos indígenas, la mayoría de las veces promotores-principales, lo cual desembocó en años posteriores en conflictos internos del municipio. Sin embargo, creemos que el INI tuvo un impacto positivo e importante: el de haber construido las fundamentos para una negociación permanente entre el Estado federal y los oxchuqueros, disminuyendo 
así el impacto de las relaciones regionales basadas en las desigualdades raciales y sociales.

Este estudio sólo da cuenta de lo que pasó en un municipio, que además resultó ser el más participativo de Los Altos. Para explicar las diferencias entre los éxitos y los fracasos del INI en la región, se tendría que estudiar cada municipio de manera independiente y enfocarse en sus dinámicas internas, lo que permitiría establecer criterios de comparación adecuados y significativos. Además, investigaciones en las instituciones nacionales y estatales, como en los archivos judiciales, permitirían verificar cuáles han sido las ventajas reales obtenidas por los indígenas, así como el control que han proporcionado dichas instituciones a las autoridades gubernamentales sobre las municipalidades indígenas. Tales investigaciones permitirían obtener un mayor entendimiento del impacto del INI en las comunidades indígenas de Los Altos de Chiapas.

\section{Notas}

${ }^{1}$ Las cuatro monografías son las de: Wasserstrom (1983), Favre (1984), García de León (1985) y Reiffer (1981).

${ }^{2}$ Esa proporción se calculó a partir de los informes del Centro que se encuentran en el Archivo Histórico del CCITT. Para mayores detalles ver Corbeil (2006: 51-57). ${ }^{3}$ En Ulrich Köhler (1975: 195, 211 y 329) consta que, si los promotores tseltales sólo eran un 30\% de los efectivos del Centro (31 tsotsiles por 15 tseltales) en 1951, eran mayoría en 1963, representando 65\% de los promotores del Centro (44 tsotsiles por 66 tseltales). Aunque afirma que esta subida en el porcentaje de promotores tseltales se debía a Oxchuc, no menciona el número de promotores originarios de este municipio. A fin de comparar, el municipio indígena más cercano a San Cristóbal, Chamula, pasó de 18 a nueve escuelas en el mismo periodo. También Romano (2002: 282) afirma que Oxchuc proporcionó el número de promotores y de alumnos más importante de toda la región del CCITT.
${ }^{4}$ Según Harman (1974: 32), la evangelización empezó en 1940 en el paraje de Yochib, al norte, y se extendió a la mitad de la población en una década. Los datos estadísticos del censo de población de 1950 muestran que no hubo ninguno evangélico en Oxchuc en esa fecha y que todos eran católicos. Sin embargo, el INI enfrentó problemas religiosos ya desde 1951 (ver AHCCITT, dirección, s/n, 1951, 5, 0090). Además, Romano (2002: 279-280) afirma que al menos un tercio de la población de Oxchuc se había convertido al evangelismo a la llegada del INI.

${ }^{5}$ Estas fuentes se encuentran en el Archivo Histórico del AHCCITT. Las referencias a éste se escriben en el siguiente formato: departamento, serie, año, caja, expediente.

${ }^{6}$ Los profesores de las escuelas federales y estatales, la mayoría mestizos o ladinos, eran mal percibidos por los indígenas porque simbolizaban la explotación que se ejercía en función de las diferencias socio-étnicas que aún existían en la región. Incluso algunos profesores solicitaron trabajo físico forzado a sus alumnos. El texto de Morales (1976: 132) constituye un buen ejemplo de esta situación.

${ }^{7}$ AHCCITT, dirección, s/n, 1951, 5, 0090.

8 Los oxchuqueros tuvieron bajas puntuales en su participación, pero se debió más a sus necesidades, como durante el periodo de escasez, que a un desafío al INI.

${ }^{9}$ Entrevista con el expromotor Pedro López Gómez, 9 de julio de 2005.

${ }^{10}$ Entrevista con el expromotor Remigio Sántiz Pérez, 24 de julio de 2005.

${ }^{11}$ Entrevista con Manuel Gómez López, 5 de julio de 2005. ${ }^{12}$ AHCCITT, dirección, informes, 1956, 2, 0016.

${ }^{13}$ Las otras dos localidades con escuela del INI, Tzunum y Tzopiljá, estaban situadas en el norte evangélico y en el sur católico, respectivamente.

${ }^{14}$ El expromotor Remigio Sántiz Pérez (entrevista del 24 de julio de 2005) confirmó este discurso, el cual se encuentra frecuentemente en los informes del Centro. 
${ }^{15}$ Daniel Gómez Rodríguez no fue promotor en esta escuela desde mayo de 1955, debido a motivos religiosos que no estaban directamente relacionados con él, sino con Mariana Slocum. De hecho, Daniel Gómez Rodríguez siguió trabajando para el INI, pero en San Cristóbal. Para el caso de Rodríguez, ver AHCCITT, dirección, s/n, 1955, 2, 0042. Para la responsabilidad del Instituto Lingüístico de Verano, ver el intercambio de cartas entre Mariana Slocum y Agustín Romano Delgado los días 28 y 31 de enero de 1955, en AHCCITT, dirección, s/n, 1955, 1, 0024.

${ }^{16}$ La escuela de Cholol tenía demasiados problemas y fue cerrada en marzo de 1957 aunque por poco tiempo, pues en 1959 un promotor enseñó nuevamente ahí. Para el caso completo, ver los documentos siguientes del AHCCITT, dirección, s/n, 1951, 5, 0090; educación, informes, 1952, 1, 0004; dirección, informes, 1956, 2, 0016; educación, informes, 1957, 1, 0003; educación, correspondencia, 1959, 1, 0008.

${ }_{17}$ En la documentación del AHCCITT, la última referencia a un conflicto religioso se encuentra en educación, correspondencia, 1959, 1, 0006. No obstante, este conflicto implicó al INI sólo por involucrar a un promotor católico que quería casarse con una mujer evangélica. Por lo tanto, el conflicto no estuvo relacionado directamente con su trabajo en el INI, sino por el hecho de que el padre de la mujer no aceptó que su yerno fuera católico.

${ }^{18}$ AHCCITT, educación, informes, 1954, 1, 0001.

${ }^{19}$ AHCCITT, educación, informes, 1952, 1, 0004.

${ }^{20}$ Estos dos conflictos aparecen en AHCCITT: dirección, informes, 1958, 2, 0022.

${ }^{21}$ AHCCITT, educación, correspondencia, 1960, 1, 0001.

22 AHCCITT, educación, informes, 1953, 1, 0007 educación, informes, 1957, 1, 0003; educación, informes, $1957,1,0003$.

${ }^{23}$ AHCCITT, dirección, s/n, 1955, 1, 0024; dirección, informes, 1956, 2, 0016 .

${ }^{24}$ Entre otros, ver AHCCITT, dirección, informes, 1956, 2,0016.
${ }^{25}$ AHCCITT, educación, informes, 1952, 1, 0004.

${ }^{26}$ AHCCITT, educación, s/n, 1956, 1, 0012.

${ }^{27}$ AHCCITT, educación, correspondencia, 1960, 1, 0001.

${ }^{28}$ AHCCITT, dirección, s/n, 1955, 2, 0037.

${ }^{29}$ AHCCITT, educación, exp. NO, 1958, 2, 0012.

${ }^{30}$ AHCCITT, educación, correspondencia, 1959, 1, 0008.

${ }^{31}$ AHCCITT, educación, informes, 1969, 7, 0183.

32 AHCCITT, dirección, informes, 1970, 4, 0081.

33 Estas cifras deben explicarse. Primero, parece sorprendente que la población total del municipio se haya triplicado en tan pocos años. Sin embargo, algunos autores confirman ese aumento radical. Entre ellos, Viqueira (2002: 222-223) afirma que, para evitar la migración en masa de los indígenas a las tierras de la clase política chiapaneca, las autoridades federales y estatales tuvieron que abrir la Selva Lacandona para la colonización ya desde los años 1940 y 1950. No obstante, el crecimiento demográfico siguió y, según el mismo autor, alcanzó en Los Altos de Chiapas un 3.7\% entre 1970 y 1990, es decir, una tasa muy superior a la media nacional de $2.6 \%$. Considerando estas cifras, se vuelve posible que la población de Oxchuc haya aumentado tanto entre 1950 y 1970. Por otra parte, se podría pensar que la proporción de $23.2 \%$ que representa la población activa en 1970, es decir 4172 personas, indica que el censo de las actividades económicas de Oxchuc se hizo de forma somera. Sin embargo, se debe considerar que la población de más de 12 años era tan sólo de 10346 personas. Si se toma en cuenta que sólo 211 mujeres fueron contadas como parte de la población activa, mientras en realidad vivían 5193 en Oxchuc, nos acercamos entonces a los números de la población activa contabilizada en el censo de 1970. Finalmente, los censos nombran otro tipo de empleos aparte de la agricultura, e indican el número de oxchuqueros que trabajaron en cada oficio. En 1950 había 3410 individuos, sobre 3465 - población activa de 12 años y más- que trabajaban en la agricultura, o sea, una proporción de $98.41 \%$. En los demás sectores, 15 trabajaban en la industria de extracción, seis en la de transformación, seis en la de construcción y 28 en 
el sector de los servicios. En 1970, 3907 personas sobre 4172 - población activa de 12 años y más - trabajaban en la agricultura, o sea, una proporción de 93.6\%. De los demás, uno trabajaba en la industria petrolera, uno en la minería, 29 en la industria de transformación, 27 en la construcción, 2 en la distribución de electricidad, 19 en el comercio, 2 en los transporte, 95 en los servicios, 10 en el gobierno, y 79 en empleos desconocidos. Como se trataba de empleos remunerados o actividades comerciales, pensamos que todos fueron considerados en el censo. Ver Secretaría de Economía, (1950 [1952]), Secretaría de Industria y Comercio, (1971) y Viqueira (2002: 223).

\section{Bibliografía}

Carmack, Robert (1989), "El impacto de la Revolución y la reforma en las culturas indígenas de los Altos: una reseña crítica de obras recientes", Mesoamérica, vol. 10 (18), pp. 401-425.

Caso, Alfonso (1955), ¿Qué es el I.N.I?, México: Instituto Nacional Indigenista.

Corbeil, Laurent (2006), Intégration nationale et éducation au Mexique. L'Instituto Nacional Indigenista dans la municipalité d'Oxchuc, Chiapas, 1951-1971, (tesis de maestría), Universidad de Montreal, Montreal.

Favre, Henri (1971), Changement et continuité chezles Mayas du Mexique, París: Éditions Anthropos.

Favre, Henri (1984), Cambio y continuidad entre los mayas de México, México: Instituto Nacional Indigenista.

García de León, Antonio (1985), Resistencia y utopía: memorial de agravios y crónicas de revueltas y profecías acaecidas en la provincia de Chiapas durante los últimos quinientos años de su historia, 2 tomos, México: Era.

Gómez Nich, Juan (1976), "Sobre el origen de la escuelaalbergue", en Aguirre Beltrán, Gonzalo, Alfonso Villa Rojas, Agustín Romano Delgado et al., El indigenismo en acción: XXV aniversario del Centro Coordinador Indigenista Tzeltal-Tzotzil, México: Instituto Nacional Indigenista/ Secretaría de Educación Pública, pp. 143-146.
Harman, Robert (1974), Cambios médicos y sociales en una comunidad maya-tzeltal, México: Instituto Nacional Indigenista/Secretaría de Educación Pública.

Henríquez Arellano, Edmundo(2000), “Usos, costumbres y pluralismo en los Altos de Chiapas”, en Viqueira, Juan Pedro y Willibald Sonnleitner (coords.), Democracia en tierras indígenas: las elecciones en los Altos de Chiapas (1991-1998), México: Centro de Investigaciones y Estudios Superiores en Antropología Social/El Colegio de México/Instituto Federal Electoral, pp. 29-60.

Köhler, Ulrich (1975), Cambio cultural dirigido en los Altos de Chiapas: un estudio sobre la antropología social aplicada, México: Instituto Nacional Indigenista/Secretaría de Educación Pública.

Lewis, Stephen (2005), The Ambivalent Revolution: Forging state and nation in Chiapas, 1910-1945, Albuquerque: University of New Mexico.

Loyo, Engracia (2006), "El conocimiento del indio. Nuevo camino para su asimilación (1930-1940)", en Bertely Busquets, María (coord.), Historias, saberes indígenas y nuevas etnicidades en la escuela, México: Centro de Investigaciones y Estudios Superiores en Antropología Social, pp. 69-94.

Modiano, Nancy (1974), La educación indígena en Los Altos de Chiapas, México: Instituto Nacional Indigenista/ Secretaría de Educación Pública.

Morales Díaz, Manuel (1976), "Mis primeras experiencias como promotor cultural”, en Aguirre Beltrán, Gonzalo, Alfonso Villa Rojas, Agustín Romano Delgado et al., El indigenismo en acción: $\chi X V$ aniversario del Centro Coordinador Indigenista Tzeltal-Tzotzil, México: Instituto Nacional Indigenista/Secretaría de Educación Pública, pp. 113-117.

Morales Sánchez, Alonso (1976), "Fundación de la escuela de Tzopiljá”, en Aguirre Beltrán, Gonzalo, Alfonso Villa Rojas, Agustín Romano Delgado, et al., El indigenismo en acción: XXV aniversario del Centro Coordinador Indigenista Tzeltal-Tzotzil, México: Instituto Nacional Indigenista/ Secretaría de Educación Pública, pp. 131-138. 
Pineda, Luz Olivia (1993), Caciques culturales (El caso de los maestros bilingües en los Altos de Chiapas), Puebla: Altres Costa-Amic.

Pineda, Luz Olivia (2004), "Maestros bilingües, burocracia y poder político en Los Altos de Chiapas", en Viqueira, Juan Pedro y Mario Humberto Ruz (eds.), Chiapas: los rumbos de otra historia, México: Universidad Nacional Autónoma de México, pp. 279-300.

Pitarch Ramón, Pedro (2004), "Un lugar difícil: estereotipos étnicos y juegos de poder en Los Altos de Chiapas", en Viqueira, Juan Pedro y Mario Humberto Ruz (eds.), Chiapas: los rumbos de otra historia, México: Universidad Nacional Autónoma de México.

Reiffer Bricker, Victoria (1981), The Indian Christ, the Indian King: The historical Substrate of Maya Myth and Ritual, Austin: University of Texas Press/Rockwell/Elsie (2006), "Historias contrastantes de la apropiación de la escritura en dos pueblos indios: los nahuas de Tlaxcala y los tzeltales de Chiapas", en Bertely Busquets, María (coord.), Historias, saberes indígenas y nuevas etnicidades en la escuela, México: Centro de Investigaciones y Estudios Superiores en Antropología Social, pp. 35-68

Romano Delgado, Agustín (2002), Historia evaluativa del Centro Coordinador Indigenista Tzeltal-Tzotzil, vol.l, México: Instituto Nacional Indigenista.
Rus, Jan (1994), "The 'Comunidad Revolucionaria Institucional': The Subversion of Native Government in Highland Chiapas, 1936-1968", en Joseph, Gilbert M. y Daniel Nugent (eds.), Everyday Forms of State Formation: Revolution and the Negotiation of Rule in Modern Mexico, Durham y Londres: Duke University Press, pp. 265-300.

Sántiz López, Marcelo (1976), "Notas autobiográficas", en Aguirre Beltrán, Gonzalo, Alfonso Villa Rojas, Agustín Romano Delgado et al., El indigenismo en acción: XX V aniversario del Centro Coordinador Indigenista TzeltalTzotzil, México: Instituto Nacional Indigenista/ Secretaría de Educación Pública, pp. 153-172.

Secretaría de Economía, Dirección General Estadística (1950[1952]), Séptimo censo general de población, estado de Chiapas, México: Secretaría de Economía, Dirección General Estadística, 6 de junio.

Secretaría de Industria y Comercio, Sección General de Estadística (1971), IX censo general de población, 1970, estado de Chiapas México: Secretaría de Industria y Comercio/Sección General de Estadística.

Viqueira, Juan Pedro (2002), Encrucijadas chiapanecas: economía, religión e identidades, México: Tusquets.

Wasserstrom, Robert (1983), Class and Society in Central Chiapas, Berkeley: University of California Press.

Womack, John, Jr. (1999), Rebellion in Chiapas: An Historical Reader, Nueva York: New Press. 
Tabla 1. Situación de empleo de los habitantes de Oxchuc en 1950 y en 1970 33

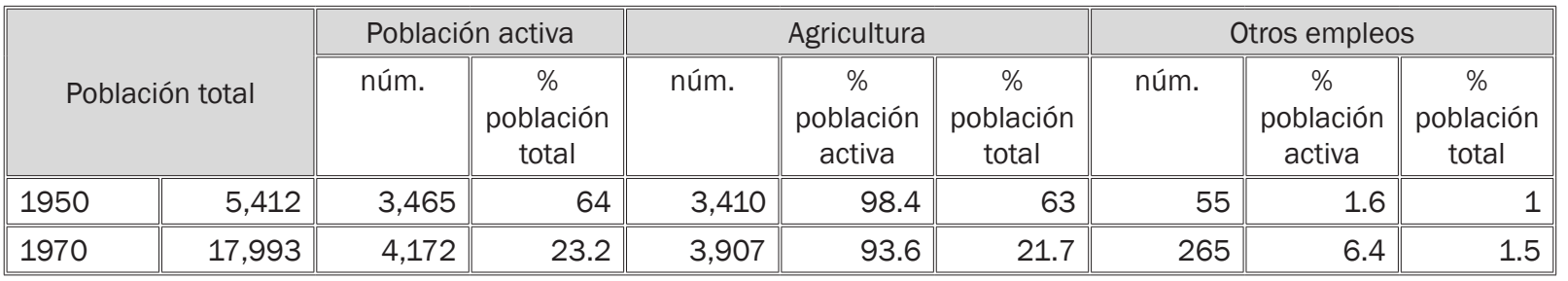

Fuente: Secretaría de Economía, (1950 [1952]), Secretaría de Industria y Comercio, (1971) y Viqueira (2002: 223). 